\title{
Mask Wearing and Interpersonal Interactions
}

\author{
DAVID B. SARWER, PHD ${ }^{1,2}$ \\ ${ }^{1}$ Department of Social and Behavioral Sciences, College of Public Health, Temple University \\ ${ }^{2}$ Center for Obesity Research and Education, College of Public Health, Temple University

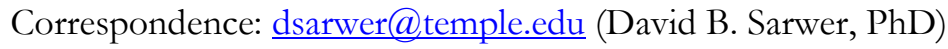

Two of the four public health pillars to reduce the spread of the Coronavirus-physical distancing and mask wearing - have clear impacts on interpersonal interactions. Evidence suggests that early physical distancing, as found by Temple University College of Public Health researchers, reduced the spread of the virus. ${ }^{1}$ The regular wearing of face coverings also is believed to have reduced spread. ${ }^{2}$ However, the universal wearing of masks has not occurred. Some areas of the country, including Philadelphia, have seen large percentages of individuals regularly wearing masks in public. ${ }^{3}$ Unfortunately, other parts of the country have not followed this lead. ${ }^{4}$ Further, some individuals have taken to openly mocking those who wear masks, even though the greatest strength of the use of face coverings is the ability to protect others from the spread of the virus if the wearer is contagious.

There is no denying that physical distancing during the pandemic has profoundly impacted interpersonal interactions. Many extended families have gone months without seeing each other in person. Seminal family events, from births to deaths, birthdays and weddings, have occurred in the absence of loved ones and close friends. Family rituals for major holidays have changed and the close friendships now occur at least 6 feet away for many. The absence of physical contact with others - the handshake, high five, and hug-is likely contributing to the negative mental health effects of pandemic. The loss of this regular physical contact is not healthy. ${ }^{5}$

The wearing of face coverings also impacts interpersonal interactions. A study that I was involved in this past summer with colleagues in the Division of Plastic Surgery at the University of Pennsylvania found that faces covered with surgical masks were rated as more attractive than those which were not masked. ${ }^{6}$ Thus, mask wearing may have its benefits- that others will find us to be more attractive. While a somewhat counterintuitive finding, we concluded that coverings on the lower face may be camouflaging more prominent facial features or asymmetries of the face that would decrease ratings of attractiveness.

While some may dismiss this study as trivial, there is a robust body of social psychological research which has developed over the past 50 years and underscores the role of physical appearance in daily life. ${ }^{7}$ There are two main findings from this scholarship. First, individuals who are rated as more attractive by others are also assumed to have more desirable personality characteristics. More attractive individuals are assumed to be more kind and honest, have more friends, and are even believed to be more intelligent. Second, numerous studies also suggest that individuals who are judged as more attractive receive preferential treatment in a wide range of interpersonal situations across the lifespan. ${ }^{7}$ More attractive children receive better grades than their less attractive peers. 
Perceived attractiveness impacts whether or not we are hired for jobs, the size of salaries, who we vote for, and how we are treated by both the legal and health care systems. It also impacts our selection of romantic partners. Thus, while we like not to admit it, our appearance matters.

This research has been summarized by the phrase "what is beautiful is good". ${ }^{8}$ There also is an often unspoken corollary - "what is not beautiful is bad". A much smaller body of research than that which supports the beauty bias suggests that individuals who are less attractive, particularly those who were born with or have acquired a facial deformity, are judged far less favorably by others. ${ }^{9}$ Those individuals who live with disfigurement, or other forms of physical disability, are often ignored by others; some are stigmatized or discriminated against as a result of their appearance. ${ }^{10}$ For many, these experiences contribute to low self-esteem, anxiety, social isolation, and depression. ${ }^{11}$ These are highly important, yet often overlooked, challenges for persons who live with physical differences in all forms.

The utilization of physical distancing to reduce the possible spread of the virus also has led to changes in health care delivery that may endure beyond the development and utilization of effective vaccines and treatments. The need to protect patients and providers from the spread of the virus led to a rapid increase in the use of telemedicine for the delivery of health care by almost every medical specialty. Some fields have long advocated for the increased use of telehealth platforms for the delivery of continuous care. The delivery of mental health care and behavioral health counseling have long seen the promise, but failure to resolve the reimbursement models for this approach to care delivery have stifled wide-spread adaptation.

The pandemic forced providers and payers to re-evaluate telemedicine almost immediately. The care of persons with clinically severe obesity presenting for bariatric surgery is a compelling example. Prior to surgery, patients are required to attend approximately 8 in person consultations with various members of the multidisciplinary team. ${ }^{12}$ Most patients complete these visits within about a 6-month period. This represents a substantial time and, in some cases, financial commitment. While some visits are relatively brief, the time traveling to and from the clinic is a significant, but often overlooked, barrier to surgery. In addition, the need to expend personal time from work, the possibility of lost wages for hourly workers, and costs associated with childcare, parking, and co-payments are largely ignored by providers, but certainly not patients. When patients have missed these visits, the multi-disciplinary team would often interpret this behavior as a lack of commitment to the program, rather than recognize that competing, relevant demands limit more complete engagement in care.

The pandemic led many programs to hold these consultations via telehealth. While almost all providers would prefer to see their patients in person, the increased utilization of telehealth visits has been accompanied by a dramatic decrease in cancellations and no shows of schedule appointments. As a result, many providers now appreciate that previously missed appointments were not a sign of ambivalence toward going forward with bariatric surgery, but for many patients, were likely the result of a lack of resources that would allow them to otherwise spend hours dedicated to an in person visit. As loss to follow up is a significant problem for patients who undergo bariatric surgery, and is believed to contribute to suboptimal outcomes, the use of telehealth to deliver targeted interventions to promote long term success holds great promise. ${ }^{13}$

Providers have learned other lessons about the delivery of care. A colleague of mine is plastic surgeon at a major health system in the Midwest. Earlier this spring, he told me that his surgeon colleagues went from 0 telehealth visits in January to over 750 in April. When I asked him what was the biggest thing that he learned during the transition to telehealth, he indicated that he realized 
that, for the most part, he often didn't need to touch his patients' wounds or scars to assess the severity of their injury or recovery from surgery. While surgeons, dermatologist, and gynecologists regularly touch their patients, it appears that for some healthcare providers doing so may not always be as necessary as once assumed.

While most mental health professionals are trained not to touch their patients, most also were trained in settings that used in person visits as the standard of care. In person visits allow the care provider to see the entire person, the overall presentation and dress of the individual, as well as physical gestures of the face and body that may help interpret a patient's emotional state. Mask wearing inhibits the ability to interpret the facial gestures of both patients and providers. Communication of the seven universal facial expressions - anger, disgust, fear, surprise, happiness, sadness, and contempt-involves the entire face. For example, while a genuine, large smile may be detectible via the narrowing of the eyes, a more subtle smile may not trigger the muscles around the eyes and, thus, may not be visible while wearing a facial covering. In the absence of the use of clear facial covering, the expression and interpretation of more subtle emotions will remain challenging and telehealth visits may be a more desirable form of care.

Regular practice of the four public health pillars is hard. Physical distancing and mask wearing has created artificial barriers between people that have likely increased feelings of sadness, loneliness, and social disconnection for many. Experts fear that the mental health toll of the pandemic many endure for years and all of us will likely forever mourn the milestone events and months of social connection that we have lost. However, the disruption of existing models of health care also provides us with an opportunity to re-evaluate the delivery of health care and prevention efforts. As a result, we may be able to embrace the true promise of telehealth as a way reduce barriers to active participation in ongoing care and promote greater engagement in chronic disease management.

\section{Disclosures and Conflicts of Interest}

Dr. Sarwer discloses consulting relationships with Ethicon and Novo Nordisk. Dr. Sarwer also serves as Faculty Editor of CommonHealth

\section{Funding Sources}

Dr. Sarwer currently has grant funding from the National Institute of Diabetes, Digestive, and Kidney Disease (R01-DK-108628-01), National Institute of Dental and Craniofacial Research (R01 DE026603), Department of Defense, as well as the Commonwealth of Pennsylvania (PA CURE).

\section{References}

1. Li X, Rudolph AE, Mennis J. Association between population mobility reductions and new COVID19 diagnoses in the United States along the urban-rural gradient, February-April, 2020. Prev Chronic Dis. 2020 Oct 01; 17:E118-E118

2. Lyu W, Wehby GL. Community use of face masks and COVID-19: evidence from a natural experiment of state mandates in the US. Health Aff. 2020 Jun 16; 39(8):1419-1425. https://doi.org/10.1377/hlthaff.2020.00818.

3. City of Philadelphia COVID-19 overview. https://public.tableau.com/shared/BMNFWW75K?:display_count=y\&:origin=viz_share_link\&:e mbed $=\mathrm{y} \&$ : showVizHome $=$ no. 
4. Percentage of people who report wearing a mask most or all of the time while in public, based on surveys of Facebook users. https:// covidcast.cmu.edu/?sensor=fb-survey-

smoothed_wearing_mask\&level $=$ county\&date $=20201018 \&$ signalType $=$ value\&encoding $=$ color\&m ode $=$ overview\&region $=42003$

5. Cohen S, Janicki-Deverts D, Turner RB, Doyle WJ. Does hugging provide stress-buffering social support? A study of susceptibility to upper respiratory infection and illness. Psychol Sci. 2015 Feb;26(2):135-47. doi: 10.1177/0956797614559284.

6. Patel V, Mazzaferro DM, Sarwer DB, Bartlett SP. Beauty and the Mask. Plast Reconstr Surg Glob Open. 2020;8(8):e3048. Published 2020 Aug 10. doi:10.1097/GOX.0000000000003048

7. Sarwer DB, Spitzer JC. Psychological Considerations in Aesthetic Surgery. In Nahai F. et al. The Art of Aesthetic Surgery: Fundamentals and Minimally Invasive Surgery, Third Edition - Volume 1. 2020.

8. Eagly AH, Ashmore RD, Makhijani MG, Longo LC. What is beautiful is good, but...:A meta-analytic review of research on the physical attractiveness stereotype. Psycholo Bulletin. 1991; 110(1):109-128. https://pdfs.semanticscholar.org/7513/4f5c90ec8f0399beac412d1d694e250be17b.pdf.

9. Jamrozik A, Ali MO, Sarwer DB, Chatterjee A. More than skin deep: Judgments of individuals with facial disfigurement. Psychology of Aesthetics, Creativity, and the Arts. 2019; 13(1):117-129. http://dx.doi.org/10.1037/aca0000147.

10. Sarwer DB, Bartlett SP, Whitaker LA, Paige KT, Pertschuk MJ, Wadden TA. Adult psychological functioning of individuals born with craniofacial anomalies. Plast Reconstr Surg. 1999; 103:412-418.

11. Rumsey N, Clarke A, White P. Exploring the psychosocial concerns of outpatients with disfiguring conditions. J of Wound Care. 2003; 12:247-252.

12. Tewksbury CM, Wu J, Allison KC, Gardiner H, Dumon KR, Williams NN, Sarwer DB: Pre-bariatric surgery care and postoperative outcomes: Increased number of visits associated with smaller weight losses over first 2 postoperative years Surgery for Obesity \& Related Diseases. Surg Obes Relat Dis. 2019 Sep;15(9):1548-1533. doi: 10.1016/j.soard.2019.06.015.

13. Bradley, LE et al Remote assessments and behavioral interventions in post-bariatric surgery patients. Surg Obes Relat Dis. 2018 Oct 1; 14(10):1632-1644. https://doi.org/10.1016/j.soard.2018.07.011

\section{ORCID ID}

David B. Sarwer, PhD: https://orcid.org/0000-0003-1033-5528 